Supporting Information for

\title{
Phenethylammonium Functionalization Enhances Near- Surface Carrier Diffusion in Hybrid Perovskites
}

Ti Wang, ${ }^{1,2 \#}$ Yongping Fu, ${ }^{3 \#}$ Linrui Jin,,${ }^{1 \#}$ Shibin Deng, ${ }^{1}$ Dongxu Pan, ${ }^{3}$ Liang Dong, ${ }^{2}$ Song Jin, ${ }^{3 *}$ and Libai Huang ${ }^{1 *}$

${ }^{1}$ Department of Chemistry, Purdue University, West Lafayette, IN 47907, USA

${ }^{2}$ School of Physics and Technology and Key Laboratory of Artificial Micro- and Nanostructures of Ministry of Education, Wuhan University, Wuhan 430072, China

${ }^{3}$ Department of Chemistry, University of Wisconsin-Madison, Madison, WI 53706, USA

* Correspondence to: libai-huang@purdue.edu,jin@chem.wisc.edu

\# These authors contributed equally to this work 


\section{Additional discussion on the crystal growth process with mixed PEA and MA cations in the solution}

The crystal growth with mixed PEA and MA cations in the solution is quite complicated due to the complex phase diagram of 2D RP perovskites and the interplay between thermodynamic and kinetic factors. The molar ratio of PEA to MA cations (defined as the $\alpha$ value) plays an important role in the crystal growth (see Figure S1 for the schematic illustration of the different growth behaviors with varying $\alpha$ values, also reproduced below). Figure S2 shows the SEM or optical images of the nanostructure films grown with different $\alpha$ values after a reaction time of around $1 \mathrm{~h}$.

Figure $\mathrm{S} 1$ schematically illustrates the crystal growth process under various $\alpha$ ratios. At a high $\alpha$ ratio, e.g. $\alpha=0.95$, microplates of $2 \mathrm{D}$ RP perovskites with low $n$ values are synthesized. At an intermediate $\alpha$ ratio, e.g. $\alpha=0.64$, majority of the initial products are surface functionalized 3D perovskite nanostructures (i.e. nanowires and nanoplates). As the reaction time increases, the initially formed 3D perovskites gradually undergo dissolution and 2D RP perovskites crystallize. At a low $\alpha$ ratio, e.g. $\alpha=0.52$, nanostructures of functionalized 3D perovskite are synthesized. The $3 \mathrm{D}$ nanostructures do not undergo apparent dissolution process as the reaction time increases.

We categorized the growth conditions into three regimes: high $(\alpha=1.18$ and 0.95$)$, intermediate $(\alpha$ $=0.78$ and 0.64$)$, and low $\alpha$ value $(\alpha=0.52)$. At high $\alpha$ values, we found $2 \mathrm{D}$ perovskite layers with different $n$ values could nucleate and grow together. This is because more PEA cations will favor the formation of 2D perovskites but the thermodynamic stability of 2D RP perovskites with similar $\mathrm{n}$ values is very small and the nucleation barriers to these phases are close. The typical $n$ value is less than 7 as revealed by the micro-PL study in our previous paper. ${ }^{1}$ In this growth regime, the morphology of the product is flake-like, due to the intrinsic layered crystal structures of 2D RP perovskites. At intermediate $\alpha$ values, we found the crystal growth process clearly changed compared to the high $\alpha$ values. Within a short period of reaction time (e.g. a few hours), the majority products were found to be $3 \mathrm{D} \mathrm{MAPbI}_{3}$ nanostructures, and the growth of $2 \mathrm{D} \mathrm{RP}$ perovskites appeared to be kinetically suppressed (Figure S3 and S4). Another distinctive phenomenon is the occurrence of nanowire growth. As the reaction time further increased, we found the initially formed $\mathrm{MAPbI}_{3}$ nanostructures gradually dissolved and 2D RP perovskite microplates started to form. The as-formed 2D RP perovskite microplates usually contain low $n$-value perovskite layers revealed by the micro-PL study (Figure S5). Some of them can be thought as 2D/3D heterostructures, for example, the microplate 2 with red curve shown in Figure S5. From these results, we conclude that while the $2 \mathrm{D}$ RP perovskites are the thermodynamically stable products, the $3 \mathrm{D}$ perovskite is the kinetically favored product, so it appears initially and is gradually converted to 2D perovskites. During the crystal growth, the surface bound cations are in dynamic equilibrium with the free cations in the solution. In the intermediate $\alpha$-value regime the PEA cations sufficiently bind to the perovskite surface yet allow the continuous growth of $3 \mathrm{D} \mathrm{MAPbI}_{3}$. In contrast, the crystal growth of 2D RP perovskites involves self-assembly of another PEA cation, which has a higher energy barrier. Therefore, 2D RP perovskite growth could occur at higher $\alpha$ values (e.g. 0.95), while was suppressed relative to $3 \mathrm{D}$ perovskite growth at the intermediate $\alpha$ values.

The functionalized $\mathrm{MAPbI}_{3}$ samples for the carrier diffusivity study were grown with a low $\alpha$ value of 0.52 that was intentionally set just below the intermediate $\alpha$-value regime. Compared to the reference $\mathrm{MAPbI}_{3}$ nanostructures grown without any PEAI, this growth condition clearly promotes anisotropic growth of the nanowires and nanoplates (Figures S6 and S7a), indicating that the PEA cations bind to the perovskite surface. The time dependent PXRD collected on the nanostructure films (Figure S7b) show that the initial formed products were 3D perovskites. Additional diffraction peaks (indicated by * in Figure S7b) at lower angles emerged with longer reaction times. These peaks cannot be assigned to the known (PEA) $2(\mathrm{MA})_{n-1} \mathrm{~Pb}_{n} \mathrm{I}_{3 n+1} \mathrm{RP}$ structures with $n=2$ and 3 (note only these two RP perovskite crystal structures have been reported previously to check 
against). These peaks are probably from higher $n$-value RP structures, of which the crystallographic structures have not been reported yet. In this growth condition the 3D perovskite nanowires and microplates appeared quite stable in the solution as the reaction proceeded, i.e. they did not undergo apparent dissolution process. This is difference from the intermediate $\alpha$-value regime. The lower $\alpha$-value will result in higher $n$-value 2D RP perovskites in the equilibrium. As the $n$ value gets larger, thermodynamic energy difference between the 2D RP perovskites and 3D perovskite is smaller. Therefore, the driving force for the dissolution gets smaller. With these results, we can sketch the rough growth "phase" diagram as a function of $\alpha$ value and reaction time (Figure S8).

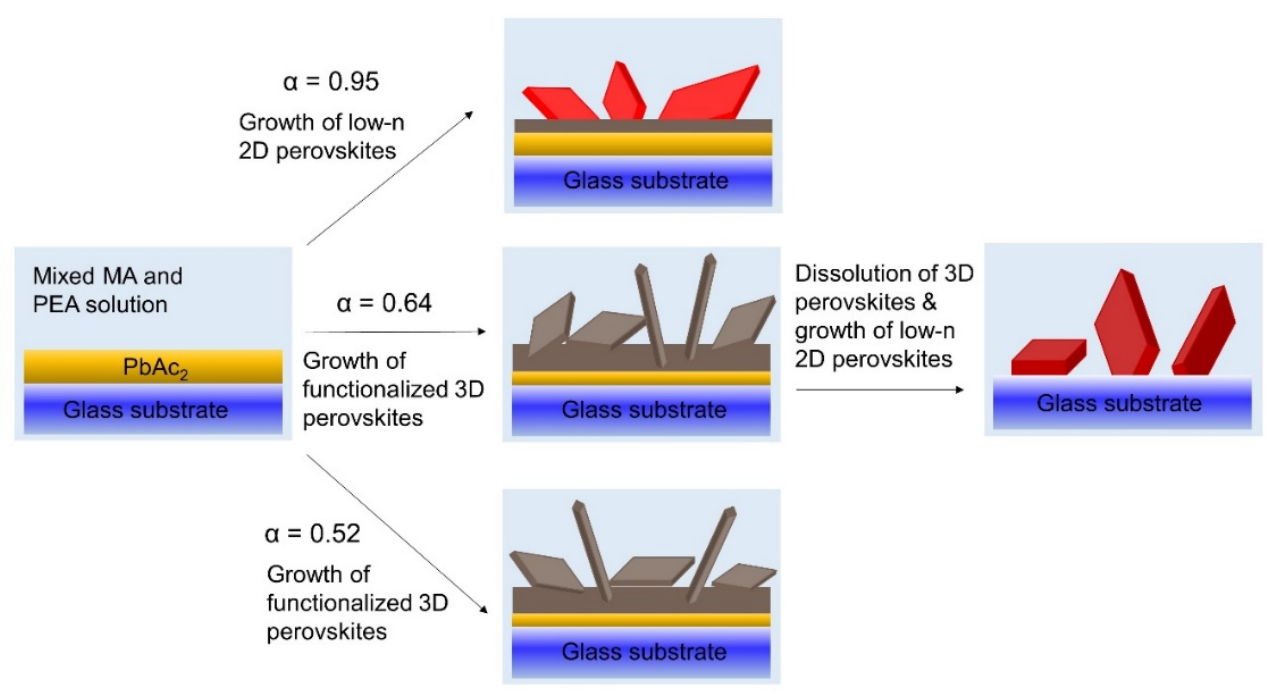

Figure S1. Schematic illustrations of the crystal growth process under various $\alpha$ ratios. At a high $\alpha$ ratio, microplates of 2D RP perovskites with low $n$ values are synthesized. At an intermediate $\alpha$ ratio, e.g. $\alpha=0.64$, majority of the initial products are surface functionalized 3D perovskite nanostructures. As the reaction time increases, the initially formed $3 \mathrm{D}$ perovskites gradually undergo dissolution and 2D RP perovskites crystallize. At a low $\alpha$ ratio, e.g. $\alpha=0.52$, nanostructures of functionalized 3D perovskite are synthesized. 


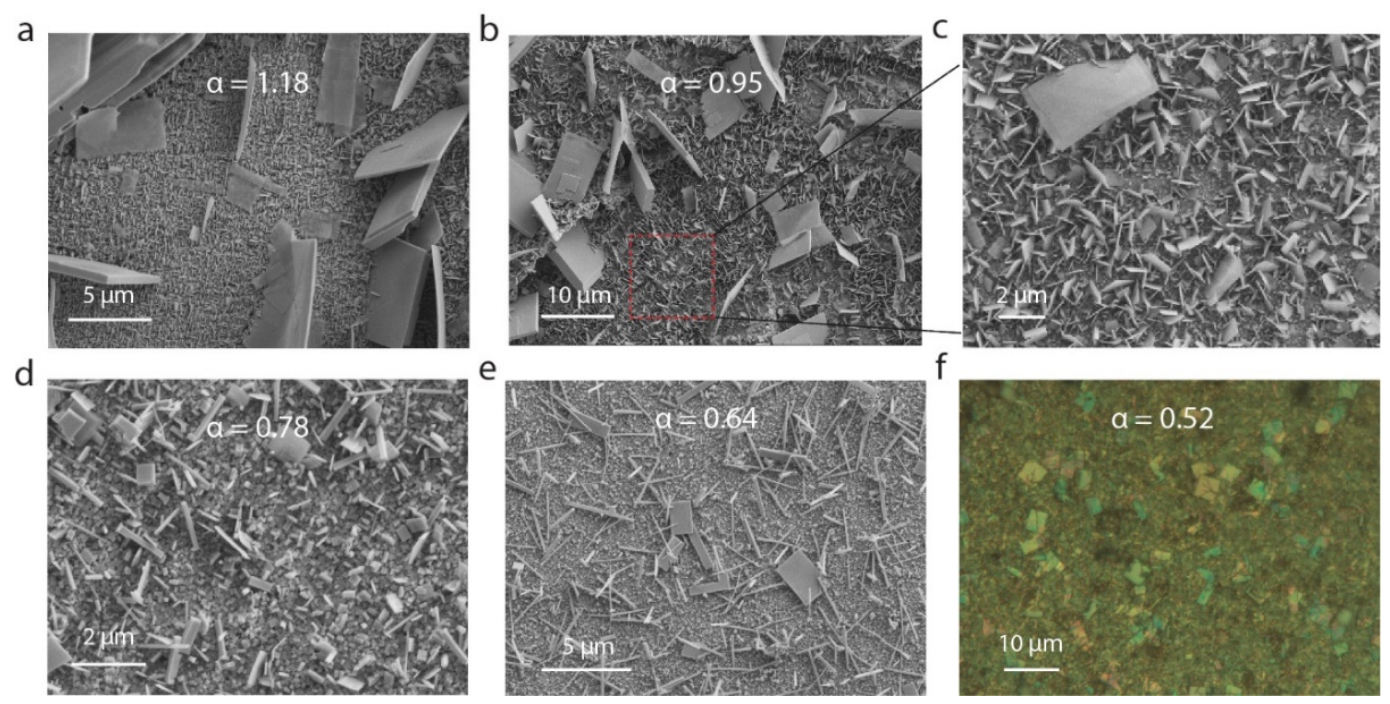

Figure S2. Scanning electron microscope or optical images of the nanostructure films grown with a $\alpha$ ratio of (a) $1.18,(b, c) 0.95$, (d) 0.78 , (e) 0.64, and (f) 0.52 after a reaction time of about $1 \mathrm{~h}$. The precursor solutions were made by mixing solutions of PEAI $(40 \mathrm{mg} / \mathrm{mL})$ and MAI $(40 \mathrm{mg} / \mathrm{mL})$ in IPA with a volume ratio of $0.65 / 0.35,0.60 / 0.40,0.55 / 0.45,0.50 / 0.50$, and $0.45 / 0.55$, respectively. At high $\alpha$ ratios, the morphology of the as-grown nanostructures is $2 \mathrm{D}$ flake-like. When $\alpha$ ratio is 0.78 or lower, nanowire morphology shows up. 


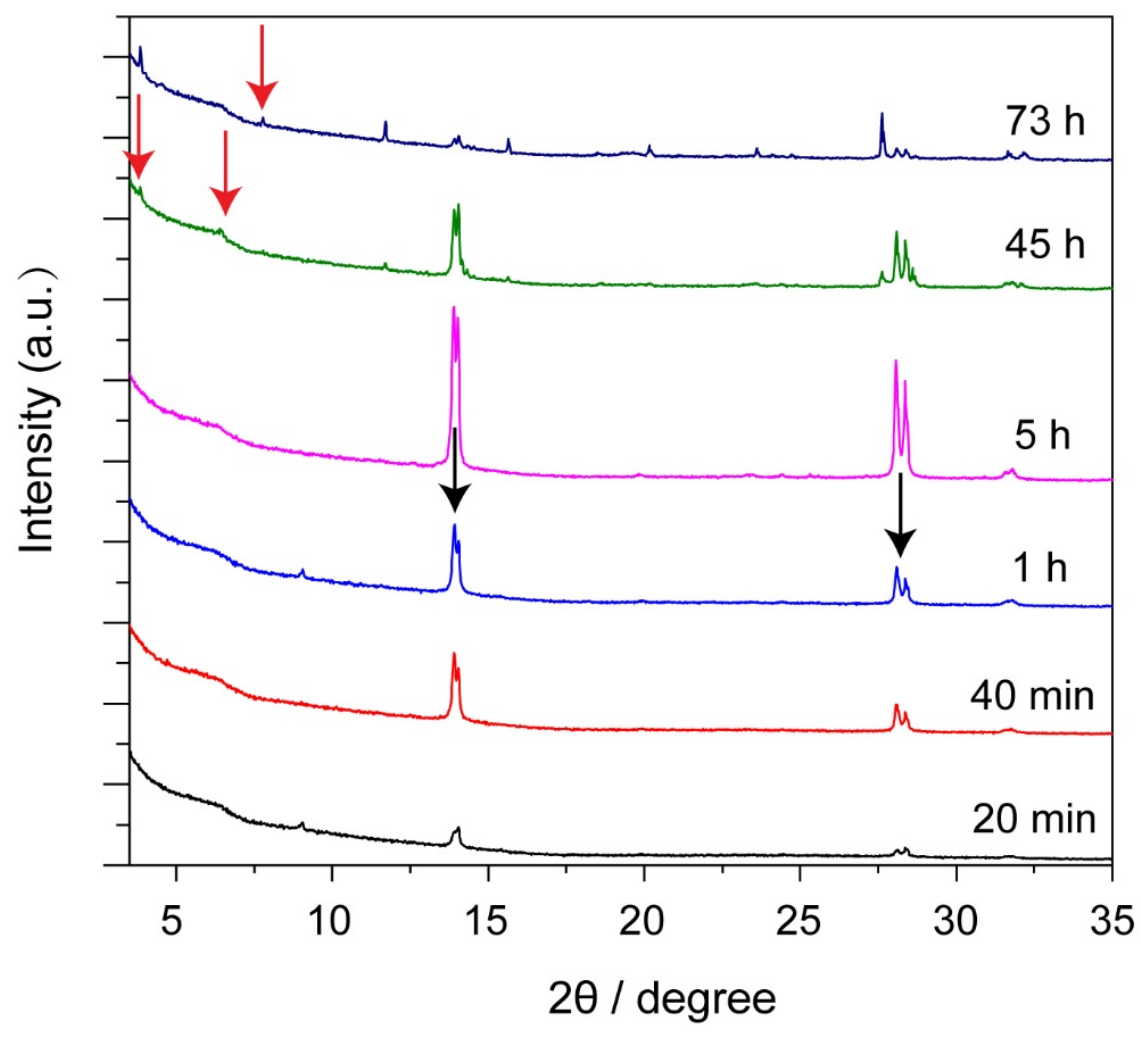

Figure S3. The time evolution of PXRD patterns of the nanostructures grown at a $\alpha$ value of 0.64 . The PXRD patterns at reaction times within several hours clearly show diffraction peaks that can be assigned to 3D $\mathrm{MAPbI}_{3}$ perovskite. Moreover, the splitting of the (110) and (002) peaks or (220) and (004) peaks (indicated by black arrows) confirms the tetragonal phase of the $\mathrm{MAPbI}_{3}$. As the reaction time goes longer, additional diffraction peaks at lower angles (indicated by red arrows) emerge and the intensities of the diffraction peaks from 3D perovskite decrease. The new lowerangle diffraction peaks can be assigned to $2 \mathrm{D}$ perovskites $(\mathrm{PEA})_{2}(\mathrm{MA}) \mathrm{Pb}_{2} \mathrm{I}_{7}$. 

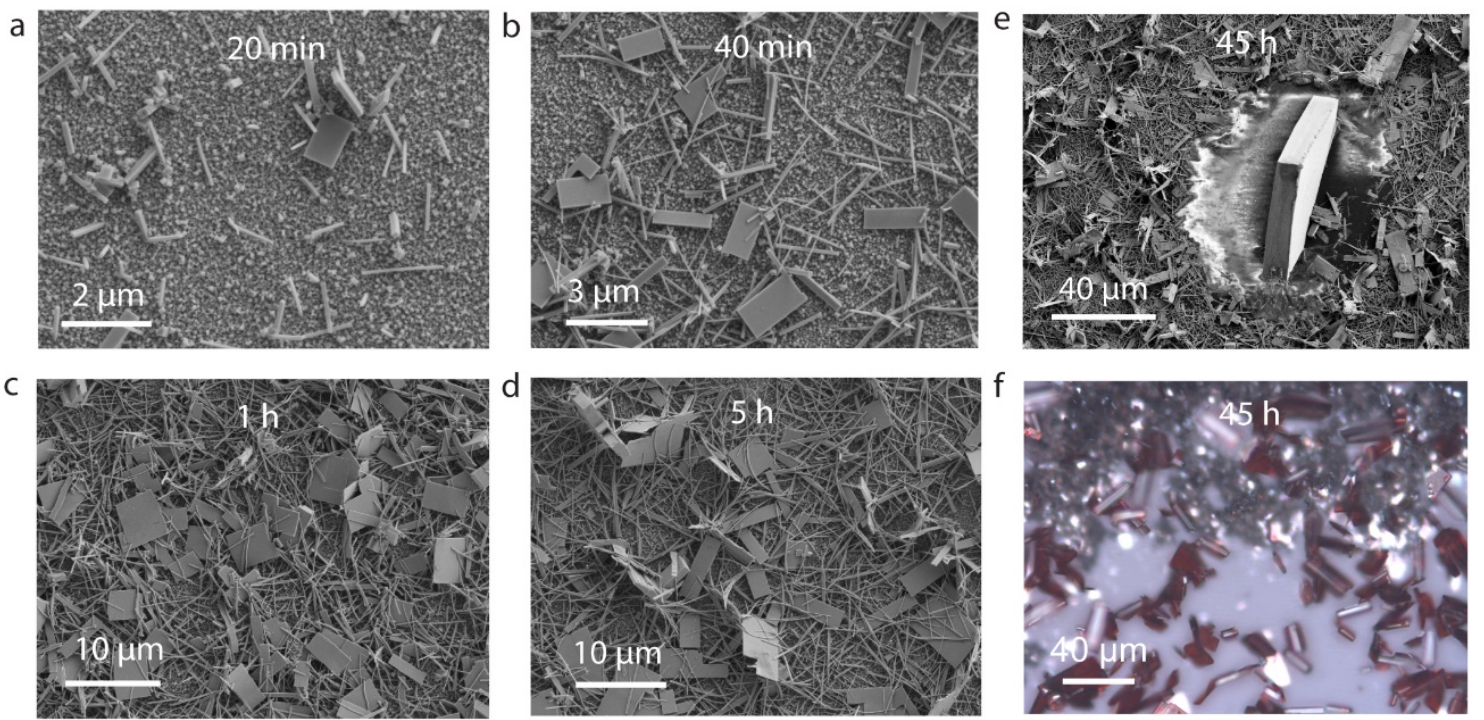

Figure S4. The corresponding scanning electron microscope or optical images of the nanostructure morphology evolution (shown in Figure S3) with reaction time at (a) $20 \mathrm{~min}$, (b) $40 \mathrm{~min}$, (c) $1 \mathrm{~h}$, (d) $5 \mathrm{~h}$, and (e) $45 \mathrm{~h}$. Within a reaction time of several hours, the products were $\mathrm{MAPbI}_{3}$ microwires and microplates. Interestingly, as the reaction time further increased, the initially formed $\mathrm{MAPbI}_{3}$ appeared to gradually dissolve and another type of large microplates formed. These microplates appear in red color under optical microscope (f), which were confirmed to be 2D RP perovskites by PL measurement (Figure S5). This is also consistently with the emerging impurity peaks in the PXRD patterns shown in Figure S3. 

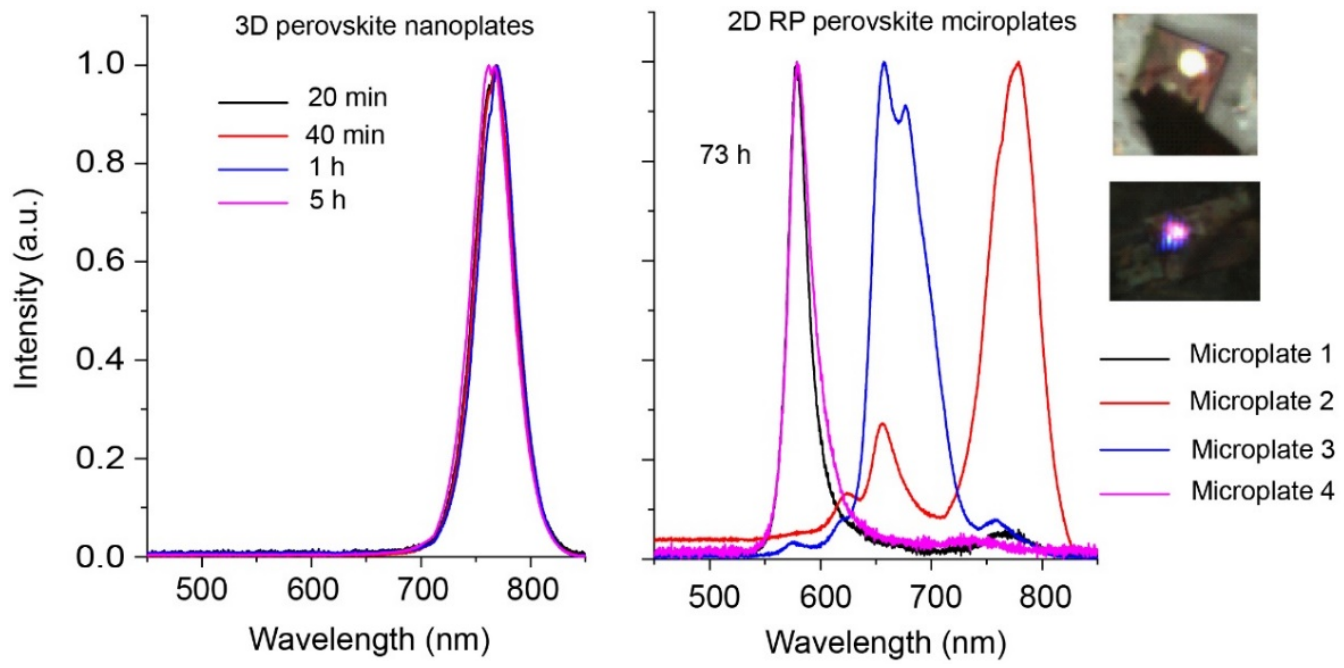

Figure S5. PL spectra of the microplates grown with $\alpha=0.64$ at different reaction times of $20 \mathrm{~min}$, $40 \mathrm{~min}, 1 \mathrm{~h}$, and $5 \mathrm{~h}$ (left panel), showing single emission peak corresponding to $3 \mathrm{D} \mathrm{MAPbI}_{3}$. In comparison, the PL spectra of several large microplates grown at a long reaction time of $73 \mathrm{~h}$ (right panel) show multiple blue-shifted emission peaks that can be assigned to the 2D RP perovskites with different $n$ values. The microplate 2 can be thought as 2D/3D heterostructure. The insets are optical images of the representative large microplates under laser excitation. These results (Figure S3-S5) indicate that the 3D perovskite is the kinetically favored product while the 2D RP phase perovskites are the thermodynamically stable products at intermediate $\alpha$ values. 


\section{a $\mathrm{MAPbl}_{3}$}
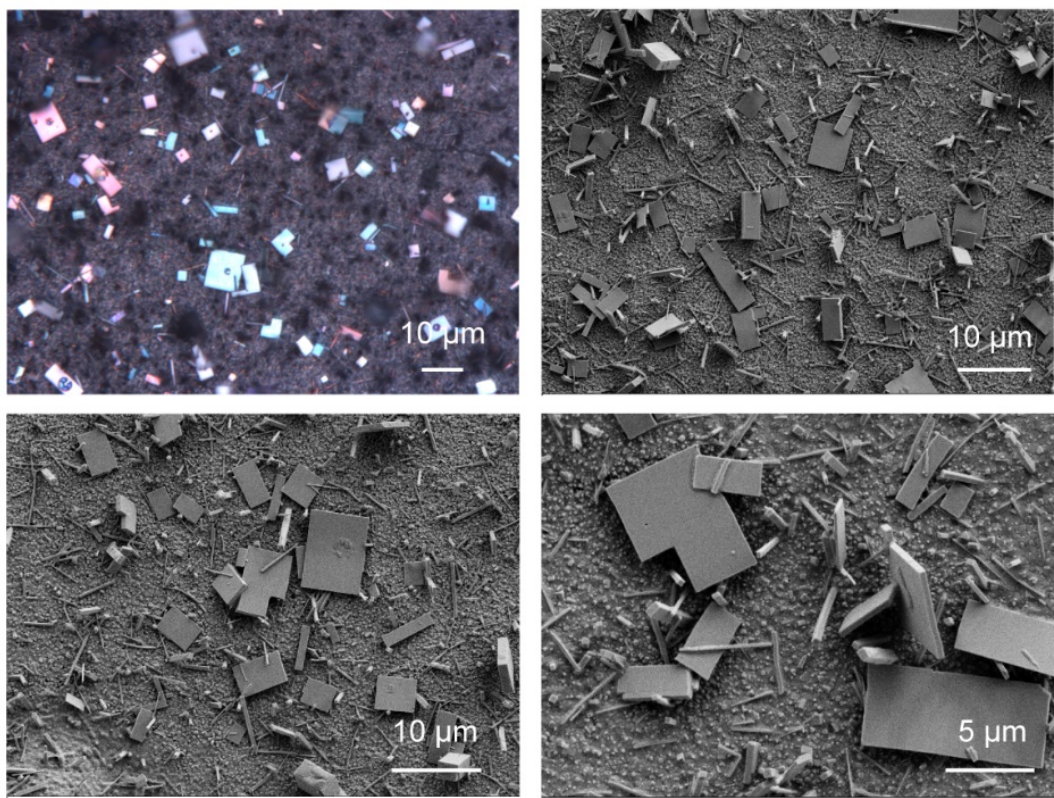

b PEA-terminated $\mathrm{MAPbl}_{3}$
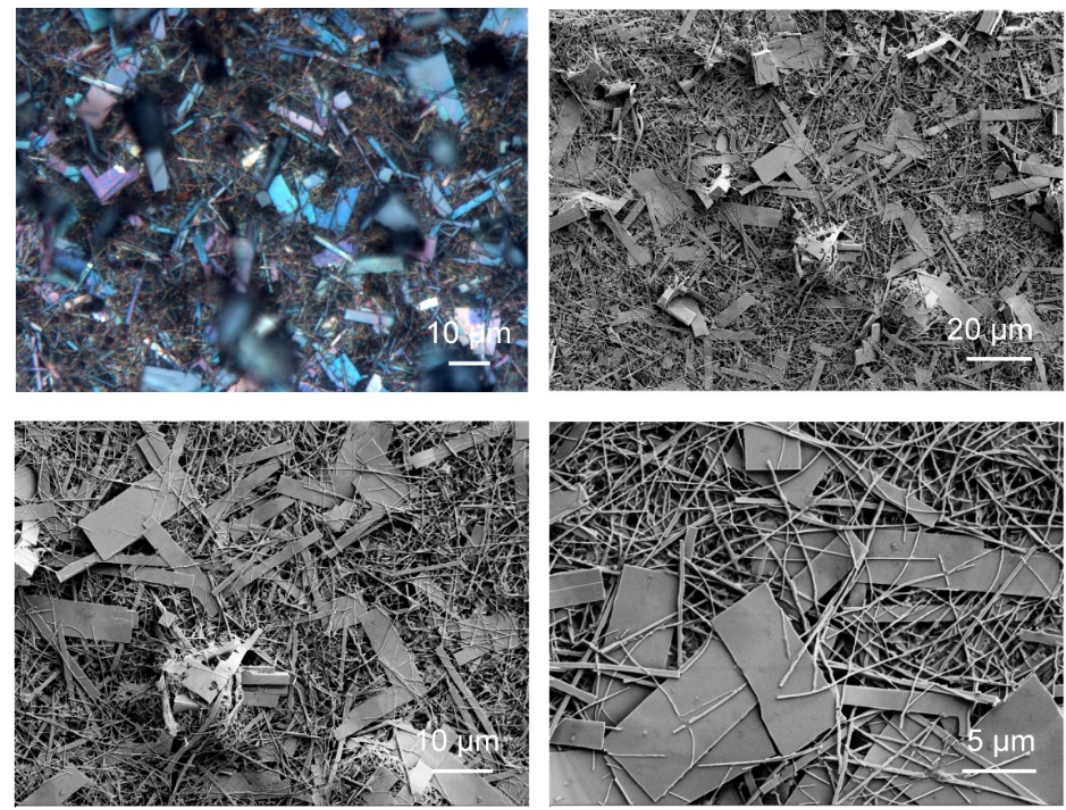

Figure S6. Optical and SEM images of (a) reference $\mathrm{MAPbI}_{3}$ microplates and (b) PEA-terminated $\mathrm{MAPbI}_{3}$ microplates, as well as some nanowires. 
a
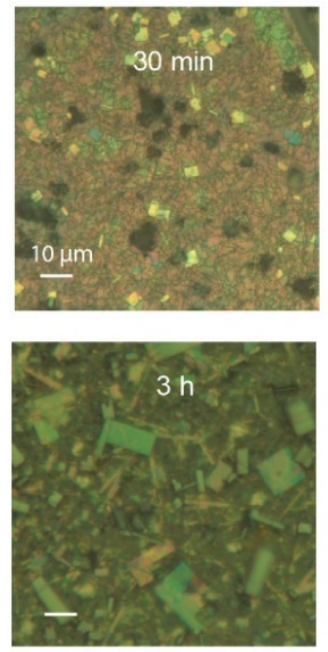
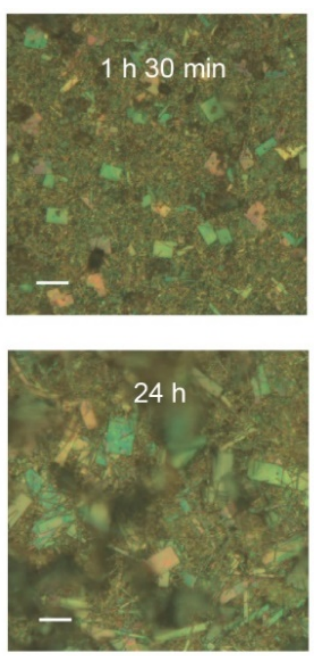

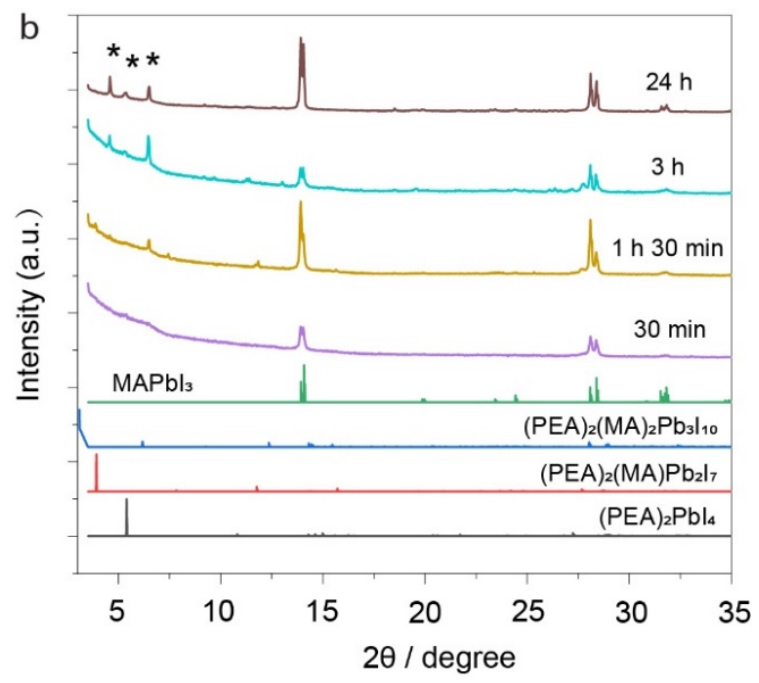

Figure S7. The time evolution of PXRD patterns and the corresponding optical images of the nanostructures grown at a low $\alpha$ ratio of 0.52 . The standard PXRD patterns for $(\mathrm{PEA})_{2}(\mathrm{MA})_{n-}$ ${ }_{1} \mathrm{~Pb}_{n} \mathrm{I}_{3 n+1}$ with $n=1,2$, and 3, and 3D tetragonal $\mathrm{MAPbI}_{3}$ perovskite are also shown for comparison. The initially formed products were 3D perovskites. Additional diffraction peaks (indicated by *) at lower angles emerged with longer reaction times. These peaks cannot be assigned to the known $(\mathrm{PEA})_{2}(\mathrm{MA})_{n-1} \mathrm{~Pb}_{n} \mathrm{I}_{3 n+1}$ RP structures with $n=2$ and 3 (note only these two crystal structures have been determined). The peaks are probably from higher $n$-value RP perovskite structures, of which the crystallographic structures have not been reported yet.

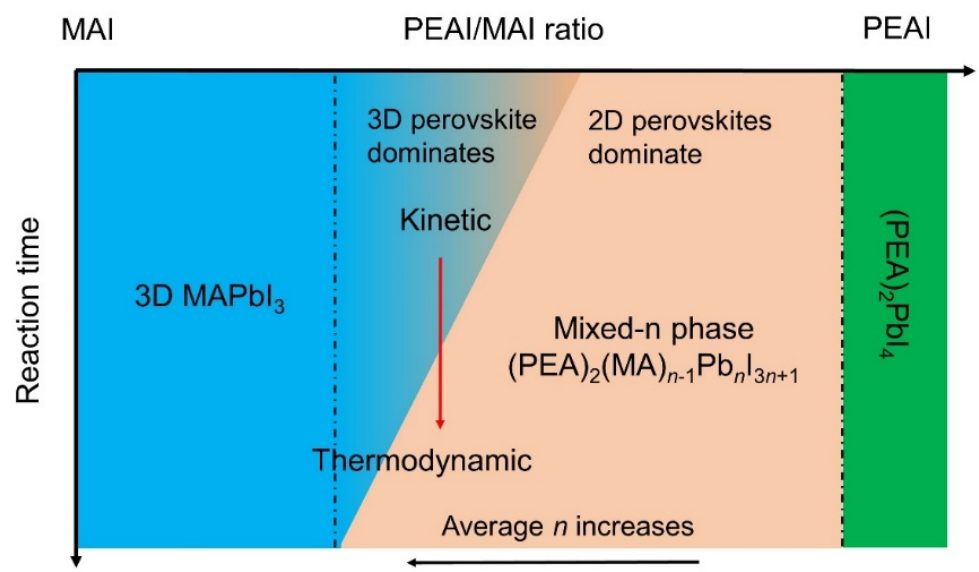

Figure S8. The proposed schematic growth "phase" diagram as a function of the $\alpha$ ratio and reaction time. In general, lower $\alpha$ ratio results in higher $n$-value 2D RP perovskites in the equilibrium. At an intermediate $\alpha$ ratio, there is an interplay between the kinetically favored 3D perovskite and the thermodynamically favored 2D RP perovskites, which makes the synthesis highly dynamic. Specifically, the 3D perovskite is the kinetically favored product during the initial period of reaction while the 2D RP phase perovskites are the thermodynamically stable products after long period of reaction time. The system will undergo dissolution of 3D perovskite and recrystallization of 2D RP perovskites. 


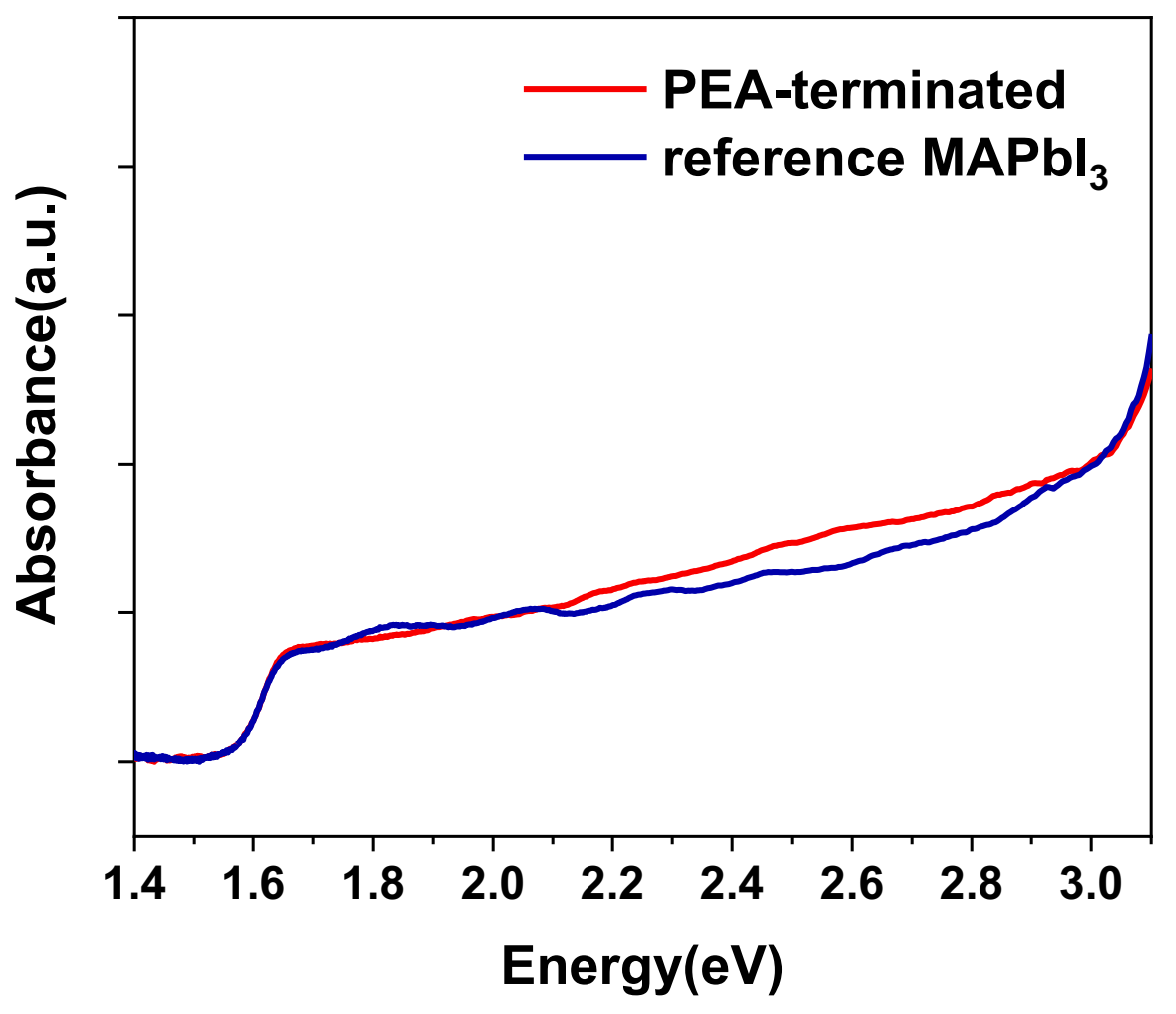

Figure S9. Absorption spectra of the reference $\mathrm{MAPbI}_{3}$ and $\mathrm{PEA}$-terminated $\mathrm{MAPbI}_{3}$ microplates. Their shapes follow the reported absorption coefficient (a) spectrum of $\mathrm{MAPbI}_{3}$ single crystals, ${ }^{2}$ using which the penetration depth of each excitation energy is calculated as $1 / a$. 


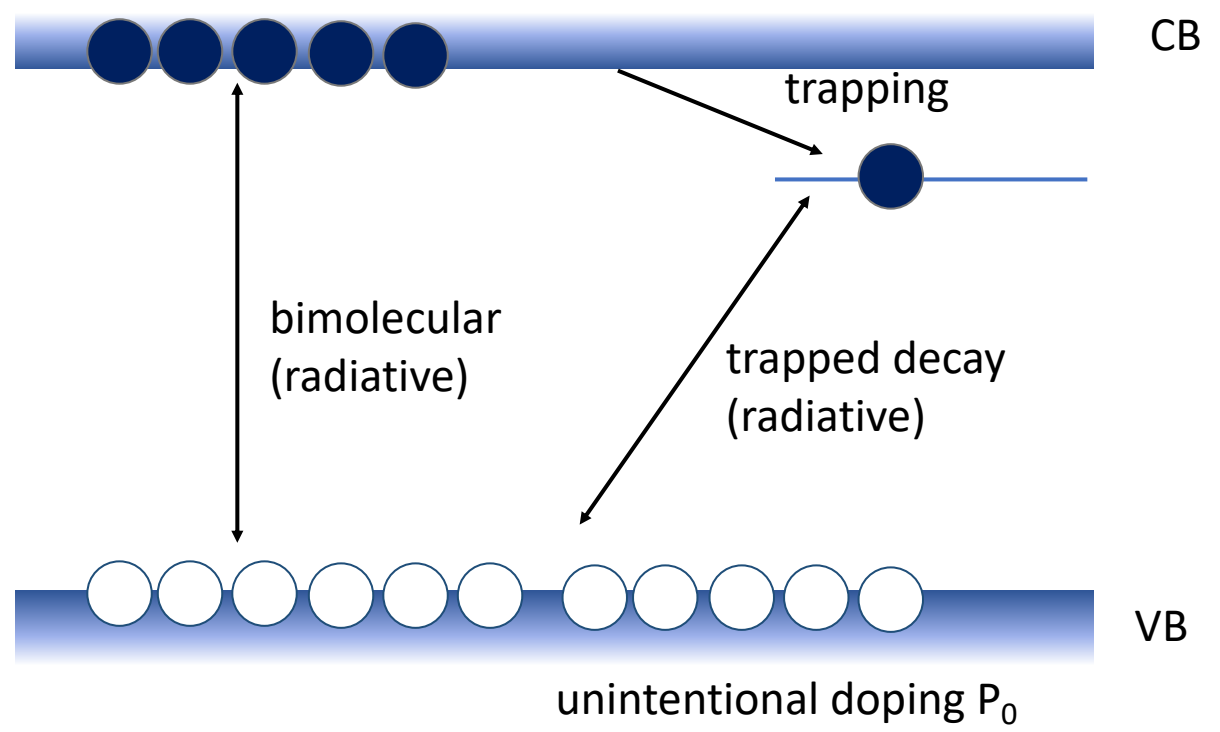

Figure S10. Schematic illustration of a trap-mediated recombination model. Here sub-gap trap states are represented with a single energy level for simplicity. The model is simplified from a reported kinetic model taking account for exciton formation, dissociation, and trapping of free electrons. ${ }^{3}$ Because of the low excitation density and small exciton binding energy $\left(\sim 12 \mathrm{meV}^{4-5}\right)$ of $\mathrm{MAPbI}_{3}$, we only consider free carriers here.

This trap-mediated recombination model gives the following rate equations

$\frac{d n_{e}(t)}{d t}=-k_{b i} n_{e}(t) n_{h}(t)-k_{t} n_{e}(t)\left(N_{T}-n_{T}(t)\right)$

$\frac{d n_{h}(t)}{d t}=-k_{b i} n_{e}(t) n_{h}(t)-k_{d} n_{h}(t) n_{T}(t)$,

$\frac{d n_{T}(t)}{d t}=k_{t} n_{e}(t)\left(N_{T}-n_{T}(t)\right)-k_{d} n_{h}(t) n_{T}(t)$,

with initial condition $n_{e}(0)=n_{0}, n_{h}(0)=n_{0}+P_{0}$, and $n_{T}(0)=0$.

Here $n_{0}$ is the initial photoexcited carrier density, $P_{0}$ is the unintentional doping density of holes, $N_{T}$ is electronic trap density. $n_{e}(t), n_{h}(t)$, and $n_{T}(t)$ are the densities of free electrons, holes, and trapped electrons. The parameters $k_{b i}, k_{t}, k_{d}$ are the rates of bimolecular recombination between free electrons and holes, trapping of free electrons to available trap sites, and depopulation of trapped electrons through holes. We assume the traps are deep enough to inhibit thermal detrapping back to the conduction band. Fitting results are shown in Figure S11 and Table S2, and sensitivity analysis to the trap density is demonstrated in Figure S12. 

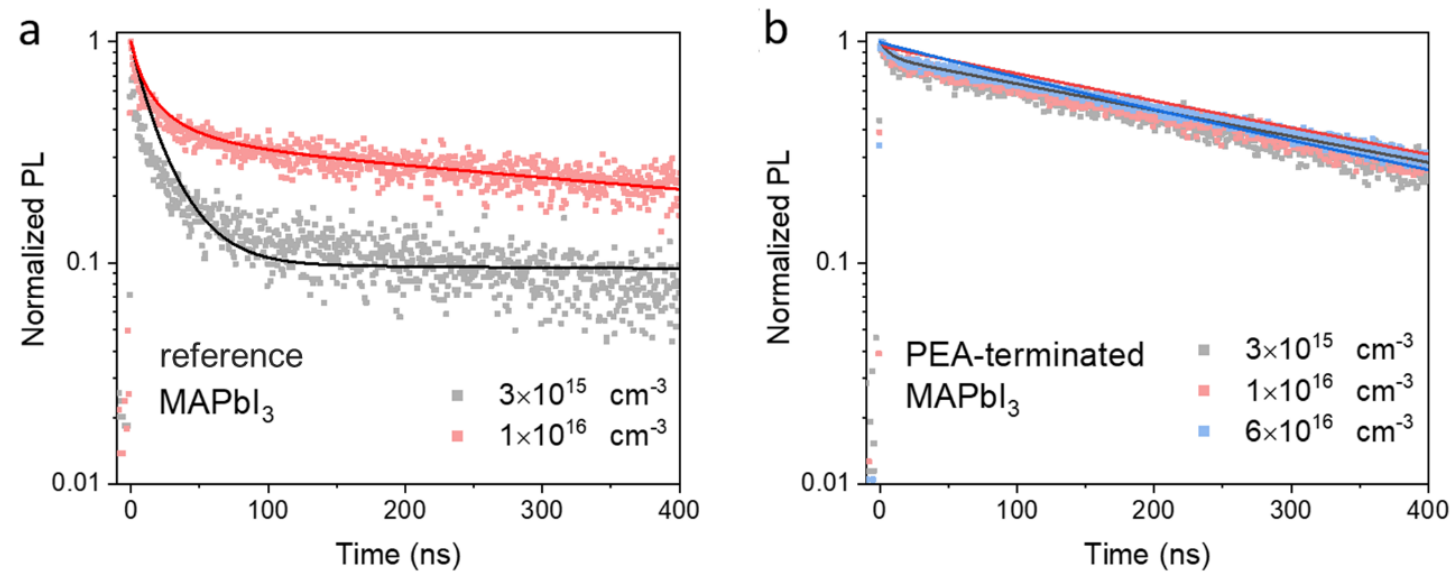

Figure S11. Carrier-density dependent time-resolved PL dynamics of (a) reference $\mathrm{MAPbI}_{3}$ and (b) PEA-terminated $\mathrm{MAPbI}_{3}$ microplates. Solid lines are the fits to the data by numerically solving the trap-mediated recombination model described in Figure S10. Fitting parameters are summarized in Table S2. Sensitivity analysis to the the trap density parameter is shown in Figure S12.

a

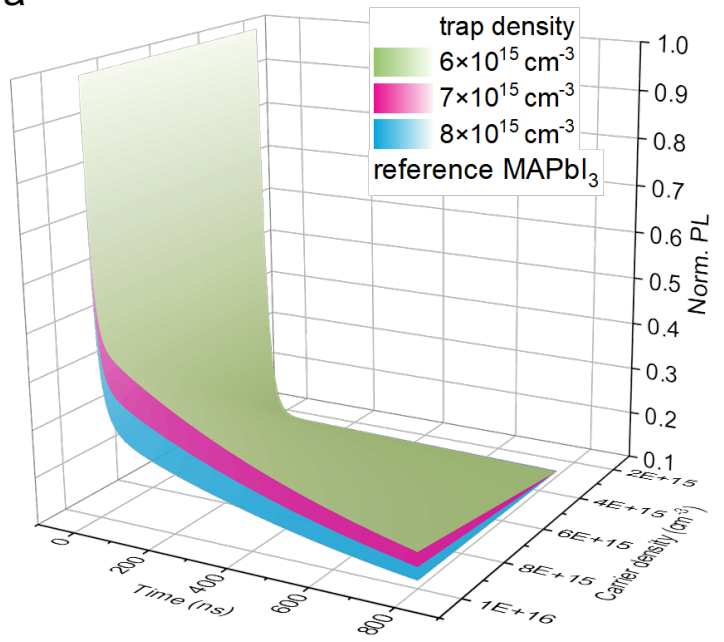

b

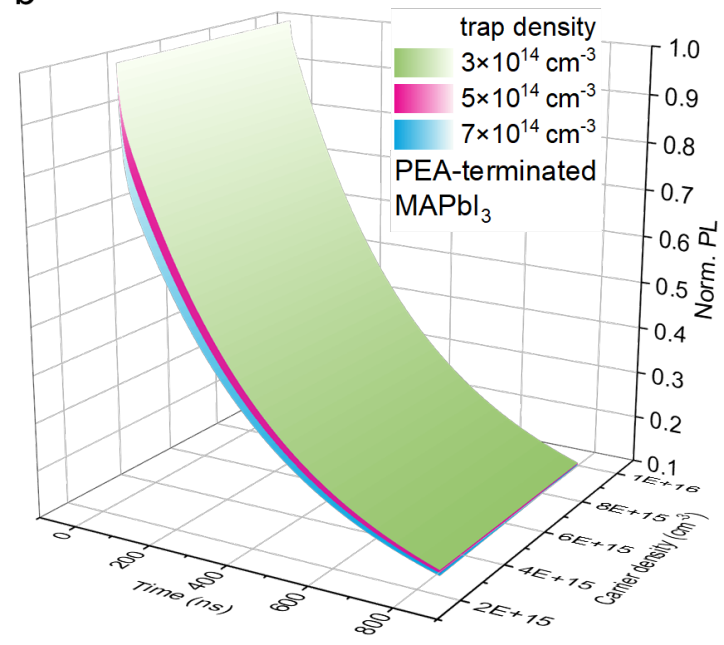

Figure S12. Sensitivity analysis of the trap-mediated recombination model (described in Figure S10) for (a) reference $\mathrm{MAPbI}_{3}$ and (b) PEA-terminated $\mathrm{MAPbI}_{3}$ microplates. Simulated PL dynamics are ploted over different initial carrier density while varying trap density around the median value. 


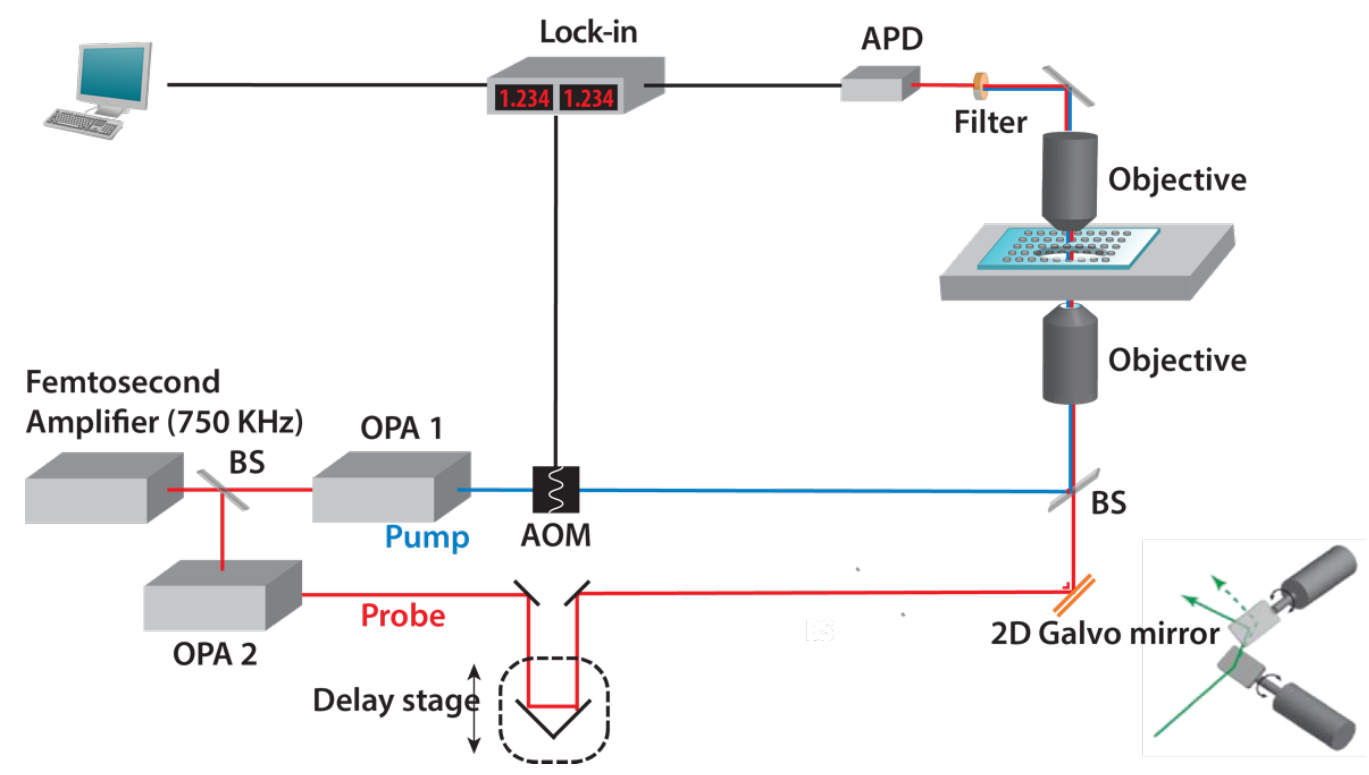

Figure S13. Schematic representation of a transient absorption microscopy (TAM) setup. The probe beam is scanned to construct images of carrier population. Abbreviations: AOM, acoustic-optical modulator; APD, avalanche photodiode; BS, beam splitter; OPA, optical parametric amplifier. 

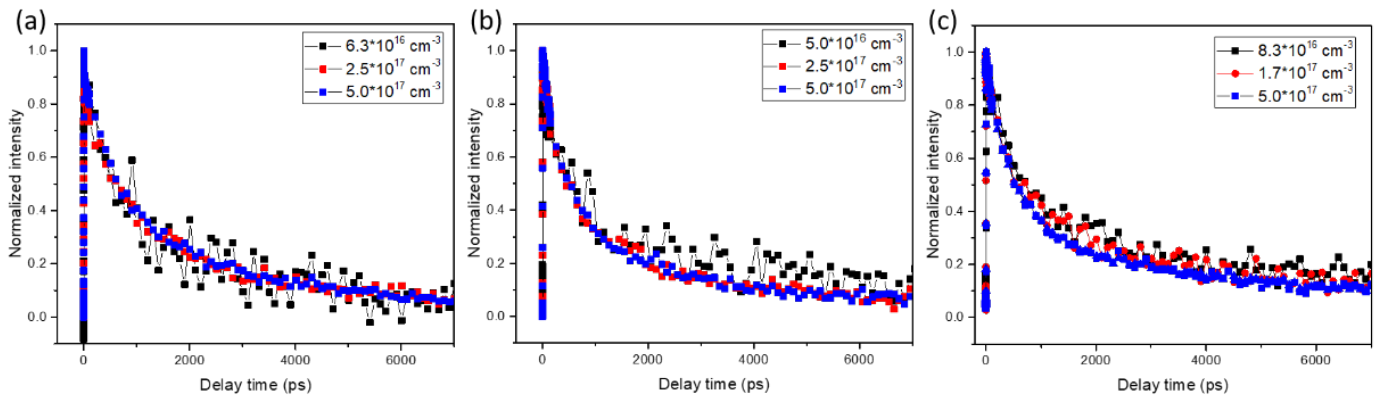

Figure S14. (a) TAM dynamics with excitation energy of $1.77 \mathrm{eV}$. (b) TAM dynamics with excitation energy of $2.25 \mathrm{eV}$. (c) TAM dynamics with excitation energy of $3.10 \mathrm{eV}$. They all show similar kinetics with various excitation densities, suggesting no annihilation in the diffusion measurements.

(a)

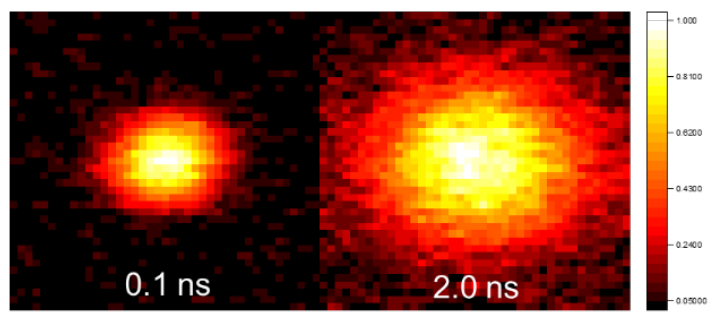

(b)

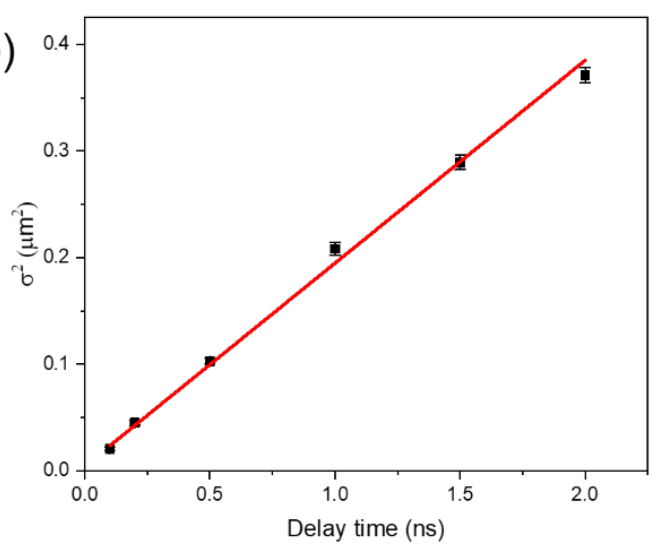

Figure $\mathrm{S} 15$. (a) The TAM images of the carrier transport at various delay times with a pump photon energy of $2.25 \mathrm{eV}$ for a reference $\mathrm{MAPbI}_{3}$ microplate. Probe energy $=1.64 \mathrm{eV}$. Color scale represents the intensity of pump-induced differential transmission $(\Delta T)$ of the probe and every image has been normalized by peak value. (b) $\sigma_{t}^{2}$ plotted as a function of pump-probe delay time. The diffusion coefficient presents a value of $0.97 \pm 0.02 \mathrm{~cm}^{2} \mathrm{~s}^{-1}$, close to the bulk-like carrier transport diffusion coefficient. 
Table S1. Bi- and mono-exponential decay fitting parameters used in Figure 2c and $2 \mathrm{~d}$.

\begin{tabular}{|c|c|c|c|c|}
\hline & $\begin{array}{l}\text { Carrier density } \\
\left(\mathrm{cm}^{-3}\right)\end{array}$ & $\mathbf{A}_{1} / \mathbf{A}_{2}$ & $\begin{array}{c}\tau_{1} \\
(\mathrm{~ns})\end{array}$ & $\begin{array}{c}\tau_{2} \\
(\mathrm{~ns})\end{array}$ \\
\hline \multirow{3}{*}{$\begin{array}{l}\text { reference } \\
\mathrm{MAPbI}_{3}\end{array}$} & $3.0 \times 10^{15}$ & 3.6 & $12.5 \pm 0.4$ & $500 \pm 26$ \\
\hline & $1.0 \times 10^{16}$ & 1.3 & $15.6 \pm 0.6$ & $850 \pm 21$ \\
\hline & $6.0 \times 10^{16}$ & 1.7 & $15.8 \pm 0.4$ & $740 \pm 16$ \\
\hline \multirow{3}{*}{$\begin{array}{c}\text { PEA- } \\
\text { terminated }\end{array}$} & $3.0 \times 10^{15}$ & 0 & - & $328 \pm 3$ \\
\hline & $1.0 \times 10^{16}$ & 0 & - & $341 \pm 2$ \\
\hline & $6.0 \times 10^{16}$ & 0 & - & $358 \pm 2$ \\
\hline
\end{tabular}

Table S2. Fitting parameters used in Figure S11 with trap-mediated recombination model.

\begin{tabular}{cccccc}
\hline & $\begin{array}{c}\text { Bimolecular } \\
\text { recombination } \\
\text { rate }\left(\mathbf{c m}^{\mathbf{3}} \mathbf{n s}^{-1}\right)\end{array}$ & $\begin{array}{c}\text { Trapping } \\
\text { rate } \\
\left(\mathbf{c m}^{\mathbf{3}} \mathbf{n s}^{-1}\right)\end{array}$ & $\begin{array}{c}\text { Trapped } \\
\text { decay rate } \\
\left(\mathbf{c m}^{3} \mathbf{n s}^{-1}\right)\end{array}$ & $\begin{array}{c}\text { Doping } \\
\mathbf{d e n s i t y} \mathbf{P}_{\mathbf{0}} \\
\left(\mathbf{c m}^{-3}\right)\end{array}$ & $\begin{array}{c}\text { Trap } \\
\text { density } \\
\left(\mathbf{c m}^{-3}\right)\end{array}$ \\
\hline $\begin{array}{c}\text { reference } \\
\mathrm{MAPbI}_{3}\end{array}$ & $4 \times 10^{-20}$ & $2 \times 10^{-17}$ & $5 \times 10^{-21}$ & $2 \times 10^{16}$ & $7 \times 10^{15}$ \\
\hline $\begin{array}{c}\text { PEA- } \\
\text { terminated }\end{array}$ & $9 \times 10^{-21}$ & $5 \times 10^{-17}$ & $1 \times 10^{-21}$ & $3 \times 10^{17}$ & $5 \times 10^{14}$ \\
\hline
\end{tabular}




\section{References Cited in the Supporting Information}

(1) Fu, Y.; Zheng, W.; Wang, X.; Hautzinger, M. P.; Pan, D.; Dang, L.; Wright, J. C.; Pan, A.; Jin, S. Multicolor Heterostructures of Two-Dimensional Layered Halide Perovskites that Show Interlayer Energy Transfer. J. Am. Chem. Soc. 2018, 140, 15675-15683.

(2) Yang, Y.; Yang, M.; Moore, David T.; Yan, Y.; Miller, Elisa M.; Zhu, K.; Beard, Matthew C. Top and bottom surfaces limit carrier lifetime in lead iodide perovskite films. Nat. Energy 2017, 2, 16207.

(3) Stranks, S. D.; Burlakov, V. M.; Leijtens, T.; Ball, J. M.; Goriely, A.; Snaith, H. J. Recombination Kinetics in Organic-Inorganic Perovskites: Excitons, Free Charge, and Subgap States. Phys. Rev. Appl. 2014, 2, 034007.

(4) Miyata, A.; Mitioglu, A.; Plochocka, P.; Portugall, O.; Wang, J. T.-W.; Stranks, S. D.; Snaith, H. J.; Nicholas, R. J. Direct measurement of the exciton binding energy and effective masses for charge carriers in organic-inorganic tri-halide perovskites. Nat. Phys. 2015, 11, 582-587.

(5) Yang, Z.; Surrente, A.; Galkowski, K.; Bruyant, N.; Maude, D. K.; Haghighirad, A. A.; Snaith, H. J.; Plochocka, P.; Nicholas, R. J. Unraveling the Exciton Binding Energy and the Dielectric Constant in Single-Crystal Methylammonium Lead Triiodide Perovskite. $J$. Phys. Chem. Lett. 2017, 8, 1851-1855. 